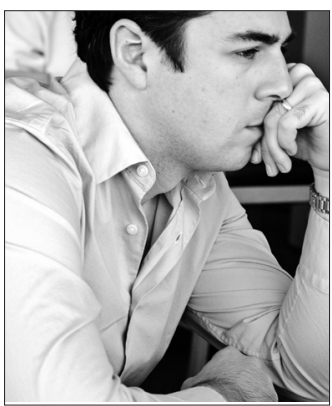

Jason Kambitsis received his MCRP from CalPoly in June 2008. He is a Senior Planner in the City of Pittsburgh Department of City Planning.

\section{Personal Digital Applications and Planning} JASON KAMBITSIS

The future of planning is here and it is largely dependent on personal digital applications. In this provocative essay, Jason Kambitsis argues that our profession must move fast to incorporate the potential of the expanding GPS technology and of portable mobile digital devices such as smart phones. Planning should become smarter and integrated to wide networks of information flows to reach a growing number of community members in their daily endeavors.

Digital applications have not always been synonymous with the planning and implementation of communities. For years planners have used stationary technologies such as maps and drawings in order to convey both onthe-ground realities and scenes from the future.

This lack of digital applications does not fully fall into the responsibility of planners underutilizing the technological advances of the past few decades. Rather, planners have been constrained by digital-divide questions that segregate community members from the planning process based upon, among others, their education and financial background. Since planning is based around the interaction between community members and professionals in a public forum, the process for gaining and displaying feedback has been constrained.

Stationary technologies that have been historically available and understood by the masses have kept planners from utilizing new technologies as they unfold. This is now starting to change. With the advent of affordable high-speed third-generation $(3 \mathrm{G})$ mobile devices and user-friendly software such as Google maps, community members of all backgrounds are beginning to get plugged into the future of planning.

Nowhere is this future more evident in smart phones applications (apps) than on CycleTracks, available on the iPhone and the Google Android operating system. This program developed by the San Francisco County Transportation Authority is designed to get real-life data from users based upon where and how they ride their bikes. It works like this. A cyclist will plug in what type of ride they are going on. They can pick from a series of options including work, pleasure, or getting groceries. From there the smart phone uses its Global Positioning System (GPS) to track the route that was taken. If the cyclist likes, he/she can send the information back to the Authority anonymously. At this point the Authority can put it into their database to get a clear understanding of the route people are taking and why. By doing this planners have the ability to gain information outside of the static public forums that are not always easily accessible to the public because of location and times. This gives planners the ability to quickly design new infrastructure or rethink how to reuse old routes for cycling.

Mass transit systems have been going through resurgence due to higher energy prices and traffic congestion attracting, among others, young tech-savvy users. These connected riders have the ability to tap into mobile messaging and GPS systems. Although the riders are quickly adopting new technologies, the transit operators have been lagging behind.

Recently, this lack of technological foresight has started to change. The biggest leap for transit connectivity comes by way of Radio Frequency Identification (RFID) systems, which have become the technology of choice. An RFID is a generic term that is used to describe a system that transmits the identity (in the form of a unique serial number of an object or person wirelessly) using radio waves." (Association for Automatic Identification and Mobility) This technology is currently being used in the Speedpass system and also ID tags for pets. 
By placing RFID chips on buses and subways the ramifications of this technology become immense. Imagine having the ability to know when your bus or subway is coming in real time. Connecting RFID to mass transit can create notification systems that send text messages to users alerting them of how quickly their ride will arrive. This will not only give transit riders the ability to plan trips more effectively, but also aid operators in designing systems more efficiently.

Mobile GPS has been used in cars for years but has only become available to the public over the last five years, destined to become as ubiquitous as the FM radio. In current form these systems give the location of the vehicle's position along with reference to where the nearest cup of coffee is or how to get to the closest hotel. Some of these systems also integrate "real time" traffic, which warns drivers of congestion and accidents.

Although these systems are becoming fully integrated between the car and driver they are not connected among all vehicles. This will soon change. Future navigation systems will not only be predicting the shift patterns of your car based on terrain but they will also have the ability to redirect your car based upon surrounding vehicle movement, and to connect with a larger transportation grid. A central transportation network will be able to instantly redirect vehicles connected to it, and also change the timing of traffic signals. By having a fullyintegrated network, the efficiency for transportation will escalate dramatically by utilizing roadways to their fullest potential, not to mention the savings in fuel that will be realized by helping to avoid traffic congestion.

These technologies are becoming the rule rather than the exception for planning. As we continue to integrate portable mobile devices such as iPhones, RFID chips, and car navigation, the field of planning has the ability to become perpetually smarter. The question is: Will planners start to capitalize on these technologies or continue down a pathway of relying solely on static maps and regimented public forums? Only time will tell, but if we do not utilize emerging technologies to their fullest potential the future may pass us by.

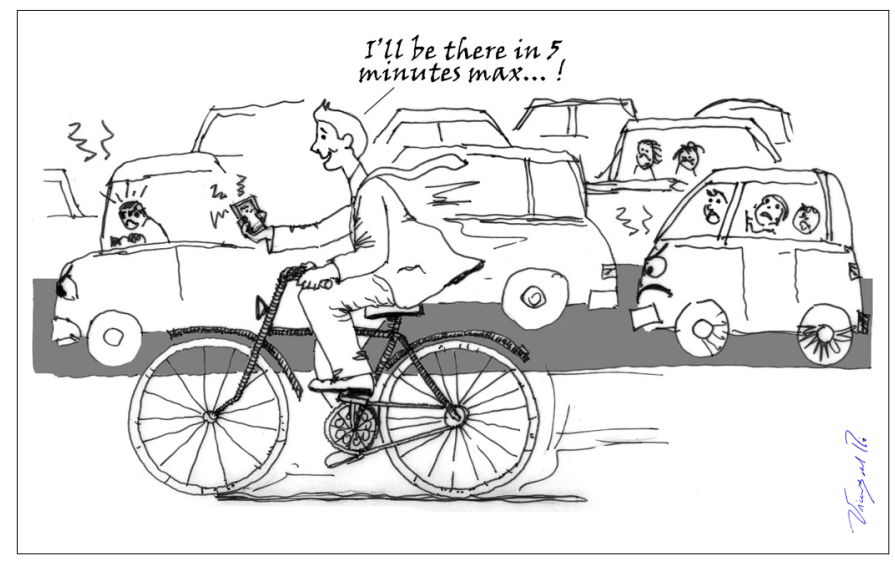

